\title{
Possible intrinsic association of anti-neutrophil cytoplasmic antibody-associated vasculitis coexisting with multiple myeloma
}

\author{
HUIFANG LIU, JIACHUAN XIONG，JUN ZHANG，YING ZHANG，LING NIE，YIQIN WANG and JINGHONG ZHAO \\ Department of Nephrology, Institute of Nephrology of Chongqing and Kidney Center of the People's Liberation Army, \\ Xinqiao Hospital, Third Military Medical University, Chongqing 400037, P.R. China
}

Received April 7, 2015; Accepted June 29, 2016

DOI: $10.3892 / \mathrm{ol} .2016 .4855$

\begin{abstract}
Anti-neutrophil cytoplasmic antibody (ANCA)-associated vasculitis (AAV) is a life-threatening condition that causes renal failure. Multiple myeloma (MM) is a malignant proliferation of monoclonal plasma cells in the blood that can also cause renal failure. The two diseases have high morbidity and mortality rates in the elderly, with a poor prognosis. A 64-year-old female presented to Xinqiao Hospital (Chonqing, China) with fatigue and a poor appetite that had been apparent for 6 weeks. Laboratory tests revealed a serum creatinine level of $10.31 \mathrm{mg} / \mathrm{dl}$, a cytoplasmic ANCA titer of 1:10, a positive result for myeloperoxidase and a serum globulin level of $3.96 \mathrm{~g} / \mathrm{dl}$. A renal biopsy revealed crescent glomerulonephritis, combined with the rapid progression of renal function. Based on these observations (ANCA titer, crescent glomerulonephritis and rapid progression of renal function) a diagnosis of AAV was established. MM was confirmed by serum immunofixation electrophoresis combined with bone marrow aspiration. The present study discusses what is, to the best of our knowledge, the first case of AAV coexisting with $\mathrm{MM}$ in order to highlight it as a clinical concern.
\end{abstract}

\section{Introduction}

Anti-neutrophil cytoplasmic antibody (ANCA)-associated vasculitis (AAV) is a life-threatening systemic autoimmune condition of unknown cause that affects small- to medium-sized blood vessels (1). AAV is comprised of three different diseases entities: Eosinophilic granulomatosis with polyangiitis, microscopic polyangiitis and granulomatosis with polyangiitis (2). Renal involvement is frequent in AAV.

Correspondence to: Dr Jinghong Zhao, Department of Nephrology, Institute of Nephrology of Chongqing and Kidney Center of the People's Liberation Army, Xinqiao Hospital, Third Military Medical University, Inpatients Building 3, 183 Xinqiao Street, Shapingba, Chongqing 400037, P.R. China

E-mail: zhaojh@tmmu.edu.cn

Key words: anti-neutrophil cytoplasmic antibody, multiple myeloma, renal biopsy, dialysis
The characteristic clinical and pathological features are rapidly progressive glomerulonephritis and pauci-immune necrotizing crescentic glomerulonephritis, respectively (3). Renal impairment has been shown to be one of the negative prognostic factors for mortality in AAV $(4,5)$.

Multiple myeloma (MM) is a malignant proliferation of monoclonal plasma cells in the blood. The most frequent complications of MM are painful pathological fractures, anemia, hypercalcemia, renal failure and recurrent bacterial infections (6). Renal impairment is a common and severe complication of MM, with an incidence rate as high as $50 \%$ (7). The United States Renal Data System showed that the 2-year all-cause mortality rate of patients with end-stage renal disease (ESRD) due to MM was 58 vs. $31 \%$ in all other patients (8). AAV and MM cause acute kidney injury via different physiopathological mechanisms, with the renal vasculature most impaired in AAV and tubular damage mainly in MM (9). A previous study reported a case in which immunoglobulin (Ig) A myeloma presented as Henoch-Schönlein purpura with nephritis (10). However, to the best of our knowledge, AAV coexisting with MM has not been previously reported. The current study presents a rare case of AAV coexisting with MM.

\section{Case report}

A 64-year-old female presented to Xinqiao Hospital (Chonqing, China) with fatigue and a poor appetite that had been apparent for 6 weeks. The patient was admitted to a local hospital 1 month previously. At this time, laboratory tests revealed a serum creatinine level of $1.33 \mathrm{mg} / \mathrm{dl}$ (reference range, $0.52-1.19 \mathrm{mg} / \mathrm{dl}$ ), a cytoplasmic (c)-ANCA titer of 1:10 (reference range, negative) and a 24-h urinary protein excretion level of $1.27 \mathrm{~g}$ (reference range, 0.00-0.12 g). The patient's creatinine level rapidly increased to $9.05 \mathrm{mg} / \mathrm{dl} 2$ weeks later and a diagnosis of acute renal failure was formed. The patient was transferred to the Institute of Nephrology of Chongqing and Kidney Center of the People's Liberation Army, Xingqiao Hospital, for further evaluation. Upon the current admission, a physical examination revealed mild elevated blood pressure $(149 / 77 \mathrm{mmHg})$ and a normal temperature of $36.5^{\circ} \mathrm{C}$. Laboratory tests showed that in a complete blood count, the white blood cell count was 2,997 cells $/ \mathrm{mm}^{3}$, the hemoglobin level was $4.3 \mathrm{~g} / \mathrm{dl}$ and the platelet count was $149,000 / \mathrm{mm}^{3}$. No microscopic hematuria was observed. Further results 
A
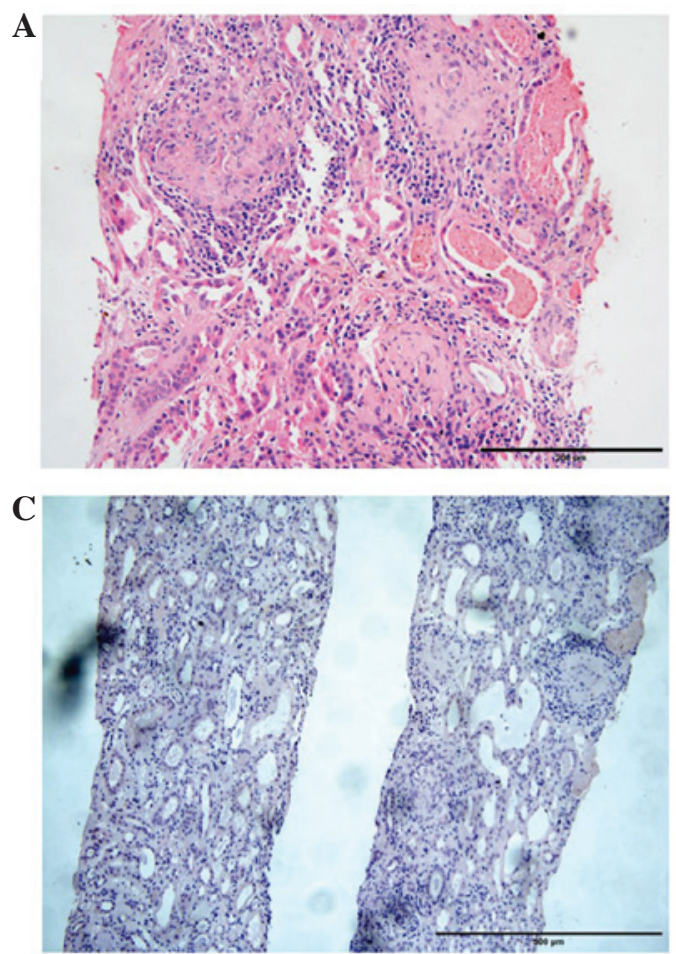

B
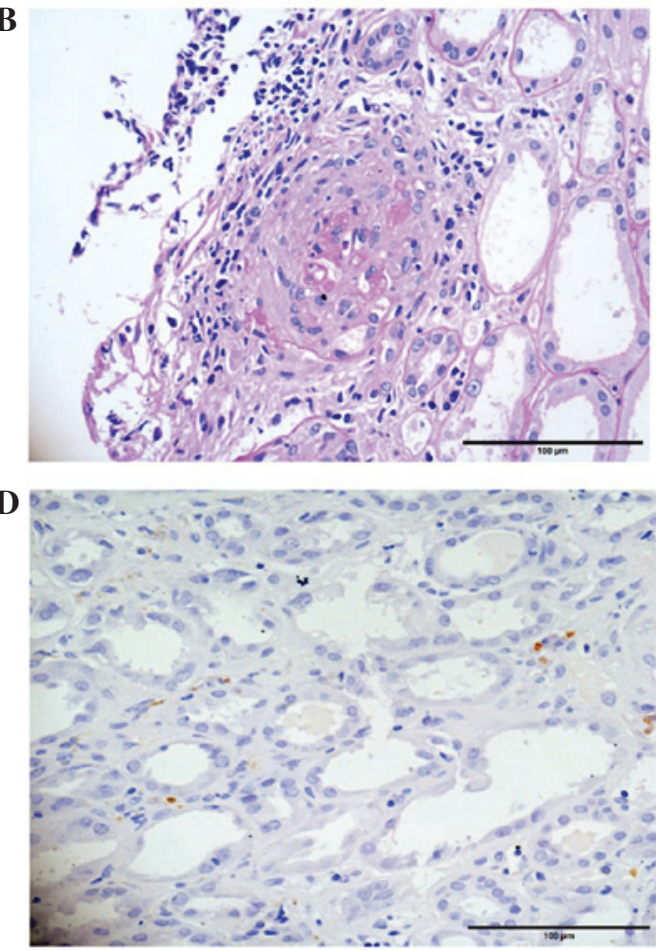

Figure 1. Renal biopsy findings. (A) A fibrocellular crescent in 1 glomerulus, diffuse sclerosis in 2 glomeruli and a red cell cast in a renal tubule cell (hematoxylin and eosin staining; magnification, x200). (B) A representative glomerulus showing a fibrocellular crescent (periodic acid-Schiff staining; magnification, $x 400)$. (C) Negative Congo red staining for amyloidosis (Congo red staining; magnification, $x 40)$. (D) Negative light chain staining ( $\kappa$ chain staining; magnification, $\mathrm{x} 400)$.

A

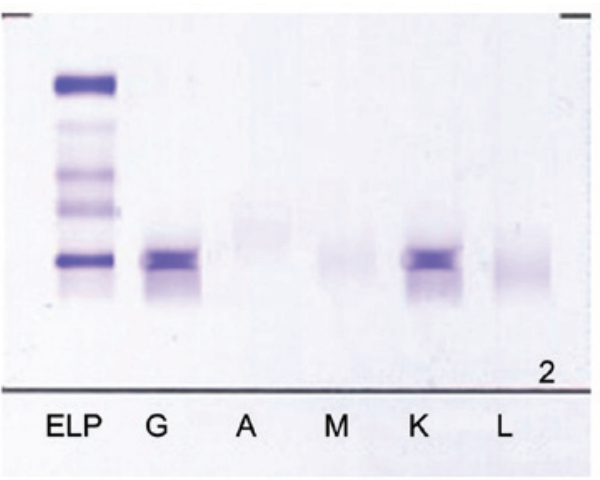

B

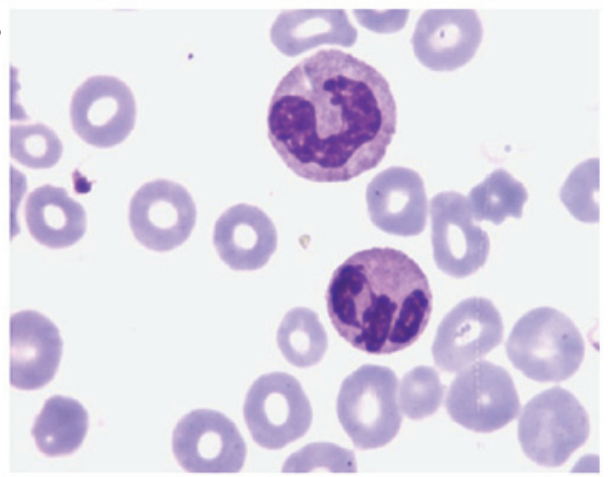

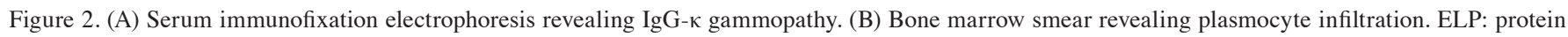
electrophoresis for control; Ig, immunoglobulin; G, IgG; A, IgA; M, IgM; K, $\kappa$ light chain; L, $\lambda$ light chain.

were as follows: 24-h urinary protein excretion, $3.5 \mathrm{~g}$; blood urea nitrogen, $99.9 \mathrm{mg} / \mathrm{dl}$; serum creatinine, $10.31 \mathrm{mg} / \mathrm{dl}$; serum albumin, $2.05 \mathrm{~g} / \mathrm{dl}$; globulin, $3.96 \mathrm{~g} / \mathrm{dl} ; \mathrm{IgG}, 2.48 \mathrm{~g} / \mathrm{dl}$; IgA, $219.00 \mathrm{mg} / \mathrm{dl}$; IgM, $106.00 \mathrm{mg} / \mathrm{dl}$; and complement 3, $0.58 \mathrm{~g} / \mathrm{l}$. The patient's c-ANCA titer was $1: 10$, the result for myeloperoxidase was positive and the antinuclear antibody titer was 1:100. The patient exhibited an elevated erythrocyte sedimentation rate of $78.0 \mathrm{~m} / \mathrm{h}$, a C-reactive protein level of $49 \mathrm{mg} / \mathrm{dl}$, and a negative result for anti-glomerular basement membrane and hepatitis B virus surface antigen. The patient was diagnosed with rapid progressive glomerulopathy and started hemodialysis immediately. Meanwhile, abdominal ultrasonography revealed that the kidneys were increased in size. A renal biopsy was undertaken carefully and 10 glomeruli were obtained; 5 glomeruli exhibited glomerulosclerosis and 1 glomerulus exhibited segmental glomerulosclerosis. A renal biopsy was carefully undertaken, and 10 glomeruli were obtained, of which, 4 glomeruli exhibited glomerulosclerosis and 1 glomerulus exhibited segmental glomerulosclerosis. Among the 10 glomeruli, 1 glomerulus was a cellular crescent, 3 glomeruli were fibrocellular crescents and 2 glomeruli were fibrous crescents. Prominent capillary loops and interstitial small artery fibrinoid necrosis were formed. Lymphocytic infiltration and fibrous tissue proliferation were observed in certain interstitial regions (Fig. 1). Renal biopsy specimens were processed for light, immunofluorescence and electron microscopy. For light microscopy, tissue specimens were fixed with $10 \%$ neutral formalin and embedded in paraffin, and $2-\mu$ m-thick serial sections were stained for hematoxylin and eosin (H\&E), periodic acid-Schiff, periodic acid-methenamine 
silver (Jones) and Masson's trichrome stains (Dako, Glostrup, Denmark). For immunofluorescence, 4-5- $\mu \mathrm{m}$-thick serial cryostat sections were stained with fluoresceinated antisera specific for IgG (cat. no. F020202-2; 1:20; Dako), IgA (cat. no. F020402-2; 1:50; Dako), IgM (cat. no. F020302-2; 1:50; Dako), C3 (cat. no. F020102-2; 1:50; Dako), C4 (cat. no. F016902-2; 1:50; Dako), $\kappa$ (cat. no. F019802-2; 1:50; Dako) and $\lambda$ (cat. no. F019902-2; 1:50; Dako) light chains. Immunofixation electrophoresis was performed by Helena Laboratories REP Rapid Electrophoresis Analyzer (Helena Biosciences, Münster, Germany). Immunofluorescence analysis noted only IgG deposition in the glomeruli, while Congo red staining and light chain staining were negative (Fig. 1). Moreover, monoclonal protein was found by serum protein electrophoresis, and confirmed by serum immunofixation electrophoresis, type $\operatorname{IgG} \kappa$ (Fig. 2). Furthermore, bone marrow aspiration was also performed and showed significantly active proliferation and plasmocytes accounting for $15 \%$ of the total cells (Fig. 2). As a result, $\mathrm{MM}$ was diagnosed. Overall, the patient was finally diagnosed with AAV coexisting with MM, based on the ANCA titer, crescent glomerulonephritis and rapid progression of renal function observed. Considering the irreversible loss to renal function and the patient's weak physical condition, regular hemodialysis was administered three times a week and arteriovenous fistula surgery was performed during the hospital stay. Other treatments included medication to control hypertension and supportive therapies. The patient was discharged The patient was discharged after 3 weeks in hospital and is followed up as an outpatient regularly every 3 months.

\section{Discussion}

To the best of our knowledge, the present study reports the first case of AAV coexisting with MM. The diagnosis of AAV was based on the serum ANCA titers and renal biopsy revealing crescent glomerulonephritis, while MM was confirmed by the serum immunofixation electrophoresis results combined with bone marrow aspiration. Although renal biopsy Congo red stain for amyloidosis was negative and immunochemistry for light chain was also negative, no abnormal plasma cells infiltration was found by $\mathrm{H} \& \mathrm{E}$ staining upon light microscopy in the renal tissue. Myeloma cast nephropathy cannot be diagnosed and excluded, as the spectrum of renal lesions in $\mathrm{MM}$ is heterogeneous, and it is undetectable in the early stage. Esnault et al (9) found that patients with monoclonal (MIg) may be positive for ANCA. While AAV is rare in hematological malignancies, Cil et al (11) detected ANCA positivity in 8 out of 60 patients with Hodgkin's lymphoma, while ANCA was not detected in non-Hodgkin's lymphoma patients (0/119). In addition, Nocente et al (12) reported a case of Sweet's syndrome associated with MIg of IgG- $\lambda$ type and perinuclear (p)-ANCA positivity, and concluded that the MIg, possibly directed to neutrophils such as an antibody, may cause their fragmentation and the release of antigens responsible for the appearance of p-ANCA. Conte et al (13) reported a case of Henoch-Schönlein purpura in a patient with MM. Henoch-Schönlein purpura is also recognized as a type of vasculitis, but without ANCA positivity. These cases lead us to believe that there is an association between the two diseases, although there is no previous direct evidence showing that $\mathrm{AAV}$ is associated with MM, although the two entities are each associated with the immune system. Thus, whether AAV and MM have an intrinsic association or whether their joint occurrence is purely coincidence requires further study.

In conclusion, AAV coexisting with MM is a rare disease and a pathological biopsy analysis is required for the diagnosis. The two disease entities are common in elderly patients and can cause acute kidney injury, progressing to ESRD, which requires kidney replacement. It appears that elderly patients should be screened for AAV and MM on the occurrence of unexplained renal failure. Meanwhile, the treatments for AAV coexisting with MM are worthy of clinical attention and further study.

\section{References}

1. Wilde B, van Paassen P, Witzke O and Tervaert JW: New pathophysiological insights and treatment of ANCA-associated vasculitis. Kidney Int 79: 599-612, 2011.

2. Jennette JC, Falk RJ, Bacon PA, Basu N, Cid MC, Ferrario F, Flores-Suarez LF, Gross WL, Guillevin L, Hagen EC, et al: 2012 revised International chapel hill consensus conference nomenclature of vasculitides. Arthritis Rheum 65: 1-11, 2013.

3. Savage CO: Pathogenesis of anti-neutrophil cytoplasmic autoantibody (ANCA)-associated vasculitis. Clin Exp Immunol 164 (Suppl 1): S23-S26, 2011.

4. Luqmani RA, Bacon PA, Beaman M, Scott DG, Emery P, Lee SJ, Howie AJ, Richards N, Michael J and Adu D: Classical versus non-renal Wegener's granulomatosis. Q J Med 87: 161-167, 1994.

5. Weidner S, Geuss S, Hafezi-Rachti S, Wonka A and Rupprecht HD: ANCA-associated vasculitis with renal involvement: An outcome analysis. Nephrol Dial Transplant 19: 1403-1411, 2004.

6. Alexanian R and Dimopoulos M: The treatment of multiple myeloma. N Engl J Med 330: 484-489, 1994.

7. Knudsen LM, Hippe E, Hjorth M, Holmberg E and Westin J: Renal function in newly diagnosed multiple myeloma-a demographic study of 1353 patients. The Nordic Myeloma Study Group. Eur J Haematol 53: 207-212, 1994.

8. Abbott KC and Agodoa LY: Multiple myeloma and light chain-associated nephropathy at end-stage renal disease in the United States: Patient characteristics and survival. Clin Nephrol 56: 207-210, 2001.

9. Esnault VL, Jayne DR, Keogan MT, Brownlee AA, Testa A, Lecarrer D, Brown DL and Lockwood CM: Anti-neutrophil cytoplasm antibodies in patients with monoclonal gammopathies. J Clin Lab Immunol 32: 153-159, 1990.

10. Zickerman AM, Allen AC, Talwar V, Olczak SA, Brownlee A, Holland M, Furness PN, Brunskill NJ and Feehally J: IgA myeloma presenting as Henoch-Schönlein purpura with nephritis. Am J Kidney Dis 36: E19, 2000.

11. Cil T, Altintas A, Isikdogan A and Batun S: Prevalence of antineutrophil cytoplasmic antibody positivity in patients with Hodgkin's and non-Hodgkin lymphoma: A single center experience. Int J Hematol 90: 52-57, 2009.

12. Nocente R, Cammarota G, Gentiloni Silveri N, Rotoli M, Zannoni GF, Bertazzoni G, Ceccanti $\mathrm{M}$ and Gasbarrini G: A case of Sweet's syndrome associated with monoclonal immunoglobulin of IgG-lambda type and p-ANCA positivity. Panminerva Med 44: 149-150, 2002.

13. Conte G, Conte FJ, Ojeda JM, Araos D, Poniachik J, Murray G and Flores C: Severe Henoch-Schönlein purpura in a patient with multiple myeloma. Rev Med Chil 128: 1255-1260, 2000 (In Spanish). 